\title{
Nikodemus Herger
}

Mit der öffentlichen Antrittsvorlesung zum Thema »Anleitung zur Unprofessionalität in der Organisationskommunikation « am 29. November 2004 hat Dr. Nikodemus Herger sein Habilitationsverfahren an der Universität Zürich abgeschlossen. Das Interesse an Vortrag, Forschungsgegenstand und an der Person war sichtlich groß.

Dr. phil. Nikodemus Herger, geboren 18.9.1960, studierte von 1983 bis 1989 Publizistikwissenschaft und Kunstgeschichte in Zürich. Nach dem Lizentiat erarbeitete er von 1991 bis 1995 berufsbegleitend die Dissertation »Private Kunstförderung als öffentlich relevantes Wirkungsfeld" (Referent: Prof. Dr. Ulrich Saxer). Angeregt durch leitende Positionen im Bereich des Kommunikationsmanagements (1997-2002 bei der Zürcher Kantonalbank und seit 2002 als Head of Corporate Branding bei der Swiss Re), befasste er sich von 1998 an kontinuierlich mit Fragestellungen der Organisationskommunikation. Seit 1999 nimmt Herger regelmäßig Lehraufträge am IPMZ - Institut für Publizistikwissenschaft und Medienforschung in Zürich wahr. Im Sommer 2004 legte er die Habilitationsschrift "Organisationskommunikation. Beobachtung und Steuerung eines organisationalen Risikos« vor. Auswärtiger Gutachter war Prof. Dr. Günter Bentele (Universität Leipzig). Vor kurzem ist die Studie unter dem gleichen Titel im VS Verlag für Sozialwissenschaften, Wiesbaden, erschienen.

Die von Herger vorgelegte Studie ist vor allem als theoretischer Diskussionsbeitrag zur laufenden wissenschaftlichen Debatte über den Status von PR und Organisationskommunikation sowohl innerhalb der Publizistikwissenschaft als auch zwischen dem Fach und den Wirtschaftswissenschaften zu sehen. Der Autor positioniert sich dabei in disziplinärer wie auch in theoretischer Hinsicht eigenständig: Er hält die in der publizistikwissenschaftlichen Tradition stehende PR-Forschung für zu verkürzt und führt deshalb das Konzept der Organisationskommunikation ein. Zugleich aber grenzt er sich mit seinem Modell deutlich ab von den in der Wirtschaftswissenschaft derzeit dominierenden Ansätzen, die PR bzw. Organisationskommunikation als Bestandteil der Kommunikationspolitik von Unternehmen auffassen. Herger versteht Organisationskommunikation als ein allgemeines theoretisches Konzept, das auch auf nicht-ökonomische Organisationen übertragen und genuin als publizistikwissenschaftliches Forschungs- und Lehrgebiet begriffen werden kann. Der Autor zeigt mit seiner empirischen Analyse (Befragung der Kommunikationsverantwortlichen großer Schweizer Unternehmen) und der darauf fußenden Zusammenfassung zudem schlüssig auf, dass Organisationskommunikation als ein eigenständiges organisationales Funktionssystem zu begreifen und innerhalb von Organisationen auch dementsprechend zu verankern sei.

Wissenschaftliche Publikationen und Vorträge liegen von Nikodemus vor allem zum Bereich Public Relations und Organisationskommunikation vor. Der theoretische Bezug und die aktuelle publizistikwissenschaftliche Diskussion werden in diesen Beiträgen stets reflektiert. Der innovative Zugriff auf das publizistikwissenschaftlich noch wenig erschlossene Teilgebiet Organisationskommunikation ist dabei hervorzuheben. Herger nimmt vom Sommersemester 2005 an seine regelmäßige Lehr- und Prüfungstätigkeit am IPMZ auf. Das Zürcher Kollegium freut sich über diese fachliche und menschliche Bereicherung.

OTFRIED JARREN

\section{Thomas Schuster}

Thomas Schuster hat sich am 20. April 2004 an der Fakultät für Sozialwissenschaften und Philosophie der Universität Leipzig habilitiert. Gleichzeitig wurde seine Lehrbefähigung für das Fach Kommunikations- und Medienwissenschaft festgestellt. Vorausgegangen waren eine Probelehrveranstaltung mit dem Thema "Narrativer Journalismus: John Herseys ,Hiroshima" "sowie der Habilitationsvortrag.

Begutachtet wurde die Habilitationsschrift "Märkte und Medien. Die Finanzmärkte im Zeitalter globaler Nachrichtennetze« von Prof. Günter Bentele, Prof. Marcel Machill (beide Universität Leip- 